\title{
A cellular automaton for the modeling of oscillations in a surface reaction
}

Cite as: J. Chem. Phys. 121, 3206 (2004); https://doi.org/10.1063/1.1770455

Submitted: 02 March 2004 . Accepted: 14 May 2004 . Published Online: 30 July 2004

M. C. Lemos, and F. Jiménez-Morales

\section{ARTICLES YOU MAY BE INTERESTED IN}

Periodical forcing for the control of chaos in a chemical reaction

The Journal of Chemical Physics 124, 014707 (2006); https://doi.org/10.1063/1.2141957

Reaction diffusion patterns in the catalytic CO-oxidation on $\mathrm{Pt}(110)$ : Front propagation and spiral waves

The Journal of Chemical Physics 98, 9977 (1993); https://doi.org/10.1063/1.464323
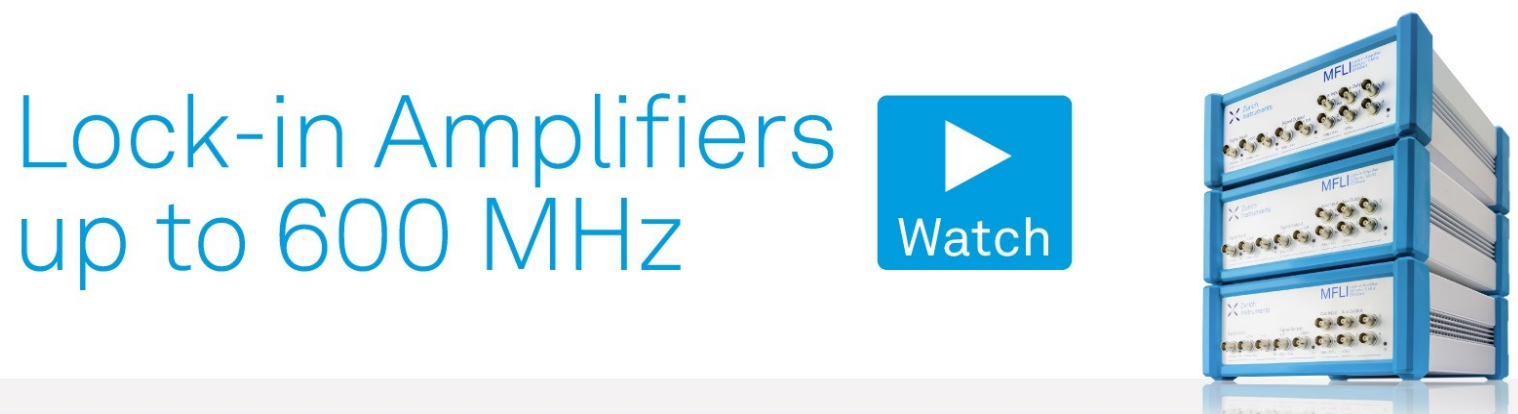


\title{
A cellular automaton for the modeling of oscillations in a surface reaction
}

\author{
M. C. Lemos and F. Jiménez-Morales \\ Departamento de Física de la Materia Condensada, Universidad de Sevilla, P.O. Box 1065, Avenida Reina \\ Mercedes s/n, 41080 Sevilla, Spain
}

(Received 2 March 2004; accepted 14 May 2004)

\begin{abstract}
The reaction of $\mathrm{CO}$ and $\mathrm{O}$ over a catalytic surface is studied with a cellular automata (CA) model. We extend the CA model proposed by Mai and von Niessen [Phys. Rev. A 44 R6165 (1991)] taking into account the variation of the temperature of the catalyst with the aim of analyzing the existence of oscillations in this reaction. The rate constants for different processes which govern the reaction are chosen in the Arrhenius form. Quasiperiodic, aperiodic, O-poisoned, and CO-poisoned regimes are observed depending on the temperature relaxation parameter. The results from the CA model presented are in agreement with several oscillatory behaviors which the catalyzed oxidation of $\mathrm{CO}$ exhibits. (c) 2004 American Institute of Physics. [DOI: 10.1063/1.1770455]
\end{abstract}

\section{INTRODUCTION}

The oxidation of $\mathrm{CO}$ by $\mathrm{O}_{2}$ over group VIII metal catalysts (Pt, Pd, or Ir) is one of the most intensely studied heterogeneous catalytic reactions. ${ }^{2-4}$ This reaction, as a good example of nonequilibrium systems, exhibits kinetic phase transitions, bistability, and associated hysteresis, and a rich variety of oscillatory behaviors, which vary from periodic to quasiperiodic and to aperiodic.

Most models proposed to describe an oscillatory behavior in the catalyzed oxidation of $\mathrm{CO}$ involve nonlinearities of different natures. For example, models, where the reaction heats generated on the catalyst may be distributed over the surface much faster than they are dissipated in the ambient and, therefore, the temperature of the catalyst can be maintained different from its surroundings, have been considered. In these models, temperature oscillations are jointly originated with concentration oscillations. Thermokinetic oscillations arise due to the highly nonlinear dependence of the reaction rate upon the temperature.

One of the first models to describe the appearance of temperature oscillations was proposed by Lagos, Sales, and Suhl. ${ }^{5}$ On the basis of mean-field theory, they obtained the kinetic equations by using the singlet closure approximation and assuming that the surface is a square lattice. They formulated the kinetics of the reaction by an autonomous system of three nonlinear differential equations in terms of the $\mathrm{CO}$ and $\mathrm{O}$ coverages, and the surface temperature. Their numerical solutions are in qualitative agreement with experiments carried out by Dauchot and Van Cakenberghe. ${ }^{6}$ The mechanism proposed by these experimenters to explain the appearance of thermokinetic oscillations is the following: (a) Adsorption of $\mathrm{O}_{2}$ is favored over that of $\mathrm{CO}$ by an increase in temperature of the catalyst. Thus, starting from the hot surface, $\mathrm{O}_{2}$ has been mostly adsorbed. (b) As the surface cools down (its surroundings are at a constant and different temperature), $\mathrm{CO}$ is progressively adsorbed and the reaction proceeds rapidly. (c) The $\mathrm{CO}$ is eliminated by the LangmuirHinshelwood (LH) mechanism and the hot surface resulting from that exothermic reaction is again covered primarily and rapidly with $\mathrm{O}_{2}$.

Lagos et al. did not consider the role played by desorption of reactives and they neglected the temperature dependence of the LH mechanism. Only the adsorption of $\mathrm{O}_{2}$ was assumed to be temperature dependent. It seems that the conditions for the occurrence of oscillations in this model ${ }^{5}$ are far from the experimental conditions, because those assumptions made such model less realistic. ${ }^{7,8}$ However, the simple structure of the model of Lagos et al. is amenable to a simple bifurcation analysis. Stability analysis of the model has also revealed that oscillatory behavior is obtained only for certain values of the parameter that controls the decay of temperature fluctuations of the surface. An interesting result obtained from their ${ }^{5}$ mathematical analysis is that, in the oscillatory state, the average yield of the reaction product may be greater than the yield in the corresponding steady state; in this way, efficiency of catalyst is improved.

In another paper, Lagos, Simoes, and Godoy ${ }^{9}$ generalize the kinetic model previously developed by them, ${ }^{5}$ incorporating an experimentally observed "slow" suboxide kinetic step. Their results are in agreement with experimental data: stable, oscillatory, and quasiaperiodic regimes are obtained.

The catalyzed oxidation reaction of $\mathrm{CO}$ was also studied by Ziff, Gulari, and Barshad ${ }^{10}$ (ZGB model). These authors studied the reaction via Monte Carlo simulations on a square lattice and found three zones with regard to the fraction of $\mathrm{CO}$ molecules in the gas phase, $y_{\mathrm{CO}}$. For low values of $y_{\mathrm{CO}}$, $y_{\mathrm{CO}}<y_{1}=0.389$, the CO pressure is low compared to the $\mathrm{O}_{2}$ pressure and, as a consequence, the catalyst is poisoned by $\mathrm{O}_{2}$. For $y_{1}<y_{\mathrm{CO}}<y_{2}=0.525$, the $\mathrm{CO}$ adsorption competes with oxygen adsorption, and the $\mathrm{CO}_{2}$ production increases with $\mathrm{CO}$ pressure. Therefore, there is a reactive steady state where $\mathrm{CO}_{2}$ molecules are continuously produced. The transition at $y_{1}$ is a second-order (continuous) kinetic phase transition. For $y_{\mathrm{CO}}>y_{2}$ the $\mathrm{O}_{2}$ pressure is low compared to the $\mathrm{CO}$ pressure, and the catalyst is poisoned by $\mathrm{CO}$. The kinetic phase transition at $y_{2}$ is of first order, because the $\mathrm{CO}_{2}$ production rate changes discontinuously.

Luque $^{11}$ studied the CO oxidation reaction on the basis 
of ZGB model. He derived the kinetic equations by starting from a master equation, following the generalized GlauberIsing model. Since the distribution of adsorbed particles on the surface can affect the transition probabilities in the adsorption and desorption processes, the spatial correlations were considered, and larger clusters that involved up to eight sites were introduced. A set of five nonlinear kinetic equations was obtained for the densities of $\mathrm{CO}\left(n_{\mathrm{CO}}\right)$ and atoms $\mathrm{O}\left(n_{\mathrm{O}}\right)$, and pairs $\mathrm{CO}-\mathrm{CO}\left(n_{\mathrm{CO}-\mathrm{CO}}\right)$, $\mathrm{CO}-\mathrm{O}\left(n_{\mathrm{CO}-\mathrm{O}}\right)$, and $\mathrm{O}-\mathrm{O}\left(n_{\mathrm{O}-\mathrm{O}}\right)$ considering a doublet closure approximation. In addition to the three zones obtained by Ziff, Gulari, and Barshad, a bistability zone was found: one reactive stable state producing $\mathrm{CO}_{2}$ and another in which the catalyst is poisoned by $\mathrm{CO}$; depending on initial conditions, the reaction will evolve towards one of the stable stationary states.

On the basis of the results obtained by Lagos, Sales, and Suhl $^{5}$ and in order to study the oscillatory behavior of the reaction, Luque's model ${ }^{11}$ was extended by us by adding an equation for the rate of change of the surface temperature. ${ }^{12}$ The results demonstrate that the nonlinearity introduced in the model gives rise to multiple stable stationary states and oscillations for certain values of the temperature relaxation parameter and other operating condition parameter. In the bistability zone, one reactive state producing $\mathrm{CO}_{2}$ and another in which the catalyst is poisoned by $\mathrm{CO}$ are found. The new reactive state can be either a stable node or an oscillatory state, and appears only for certain values of the temperature relaxation parameter. Thus, as in other models, this parameter plays an important role in the onset of oscillations.

In addition to the use of mean-field theory and Monte Carlo (MC) simulation for the study and modeling of heterogeneous catalytic systems, recently the technique of cellular automata $^{13}(\mathrm{CA})$ is another possibility to simulate the oscillatory behavior of the oxidation of $\mathrm{CO}$ on metallic surfaces with the advantage that the $\mathrm{CA}$ approach, faster than $\mathrm{MC}$ simulations, may become equivalent to the latter. A cellular automaton consists of a discrete number of cells or sites which evolve in discrete time steps following the same deterministic (or probabilistic) rules. These simple transition rules can be easily programmed. In the course of the temporary evolution each cell can only adapt values from a finite set. The rules for the evolution of a cell only depend on the state of the cells in the local neighborhood. At each time step the value of each site is updated. This feature allows parallel processing and results a substantial reduction in the computational time. Physical, chemical, and biological systems with many discrete elements and with local interactions can be modeled using CA. A survey of recent literature indicates that the study of surface chemical reactions with CA is possible. So, there is a study of the ZGB model by Mai and von Niessen. ${ }^{1}$ They introduce a CA approach which describes the steps of adsorption and reaction correctly and obtain a good quantitative agreement with the ZGB model. Later, Tambe, Jayaraman, and Kulkarni, ${ }^{14}$ following Mai-von Niessen CA model, formulate $\mathrm{CA}$ transition rules for the catalytic oxidation of CO incorporating the Eley-Rideal (ER) step, that is, the reaction between $\mathrm{CO}$ in the gas phase with the atomic adsorbed oxygen. In this paper, we extend the CA proposed by Mai and von Niessen ${ }^{1}$ in order to analyze the existence of thermochemical oscillations in the case of $\mathrm{CO}$ oxidation. In this way, we can compare the results obtained by us in a previous article ${ }^{12}$ with the ones computed here via CA simulations.

In the following section, the cellular automaton model of Mai and von Niessen is extended by adding an equation for the rate of change of the surface temperature. The results of our simulations are described in Sec. III and discussed in the light of mean-field solutions obtained in a previous paper. The conclusions and future developments of CA model presented here are summarized in the last section.

\section{THE MODEL}

The surface of the catalyst is represented as a twodimensional square lattice with periodic boundary conditions. The assumption is that the molecules of carbon monoxide $\mathrm{CO}$ (ads) and the oxygen atoms $\mathrm{O}$ (ads) both occupy single active sites on the lattice, where "ads" indicates that the species is adsorbed on the surface. The carbon monoxide and oxygen molecules, $\mathrm{CO}$ (gas) and $\mathrm{O}_{2}$ (gas), collide randomly on the surface and when there is an available site they are adsorbed. Thus, gaseous $\mathrm{CO}$ (gas) and $\mathrm{O}_{2}$ (gas) can competitively be adsorbed on the same active sites. Two adjacent sites are needed for the oxygen molecule $\mathrm{O}_{2}$ (gas) to be adsorbed. Upon adsorption, the $\mathrm{O}_{2}$ (gas) dissociates into two $\mathrm{O}$ atoms, each one residing on a separate surface site. When a molecule is adsorbed on the surface, it becomes fixed, so that superficial diffusion is ignored in this model. If a $\mathrm{CO}$ (ads) molecule is a neighbor of an $\mathrm{O}$ (ads) atom, the reaction occurs and the product molecule $\mathrm{CO}_{2}$ is desorbed as soon as it is formed. The model assumes that the reaction takes place according to three basic steps:

$$
\begin{aligned}
& \mathrm{CO}(\text { gas }) \rightarrow \mathrm{CO} \text { (ads) } \\
& \mathrm{O}_{2} \text { (gas) } \rightarrow 2 \mathrm{O} \text { (ads), } \\
& \mathrm{CO}(\text { ads })+\mathrm{O} \text { (ads) } \rightarrow \mathrm{CO}_{2} \text { (gas). }
\end{aligned}
$$

The reaction between dissociatively adsorbed oxygen and adsorbed CO, process (3), is known as the LH mechanism. The rate constants $k_{i}$, for each one of three kinetic mechanisms named above ( $i=1$, adsorption of $\mathrm{CO} ; i=2$, adsorption of $\mathrm{O}_{2} ; i=3$, desorption of $\mathrm{CO}_{2}$ ), are chosen in the Arrhenius form:

$$
k_{i}=A_{i} \exp \left(-\frac{E_{i}}{k_{B} T}\right)
$$

where $A_{i}$ are frequency factors, $k_{B}$ is the Boltzmann constant, $T$ denotes the temperature of the surface, and $E_{i}$ are activation energies of each one of the three elemental processes. These energies correspond to the action of the substrate and are assumed to be coverage independent. With the aim of studying the oscillatory behavior of the reaction, the model must be supplemented by the addition of an equation describing the variation of the surface temperature due to the adsorption and reaction processes:

$$
C \frac{d T}{d t}=-L\left(T-T_{B}\right)+\sum_{i=1}^{3} \Delta H_{i} p_{i},
$$


where $C$ is the heat capacity of the catalyst, $L$ is the heat transfer coefficient ( $L$ is approximately equal to thermal conductivity multiplied by a geometric factor), $T_{B}$ is the room temperature, $\Delta H_{i}$ are the reaction heats of the processes (1)(3), including also a suitable geometric factor, and $p_{i}$ are the number of processes of type $i$ which take place per unit area. The previous equation can be written as

$$
\frac{d T}{d t}=-\gamma\left(T-T_{B}\right)+\sum_{i=1}^{3} h_{i} p_{i},
$$

where $\gamma=L / C$ is the dissipative factor or the relaxation rate of $T$ to $T_{B}$ and $h_{i}=\Delta H_{i} / C$. Thus, the linear term $-\gamma(T$ $-T_{B}$ ) describes the dissipation heat from the catalyst surface towards its surroundings, while $\Sigma_{i=1}^{3} h_{i} p_{i}$ is the net heat generated on the catalyst surface in the reaction steps. The model can give rise to oscillatory behavior for certain values of the set of parameters $\left\{A_{i}, E_{i}, h_{i}, \gamma\right\}$, room temperature being at $T_{B}=300 \mathrm{~K}$. The kinetics of the reaction is described in terms of the density of adsorbed particles of $\mathrm{CO}$ and $\mathrm{O}$, $n_{\mathrm{CO}}$ and $n_{\mathrm{O}}$, the production of $\mathrm{CO}_{2}, R$, that is, the density of pairs $\mathrm{CO}-\mathrm{O}$ which are formed and leave the surface immediately after its formation, and, finally, the surface temperature $T$, which differs from the temperature of the gas. Let us emphasize the difference between the ambient temperature $T_{B}$, which is essentially constant in time, and the surface temperature $T$ of the catalyst. The model involves the surface temperature as a dynamical variable. The variation of this surface temperature, being subject to Eq. (4), can lead to thermal instabilities because the dependence of the different reaction steps (1)-(3) upon the temperature at Arrhenius law is the main feedback mechanism generating oscillations. We follow Mai and von Niessen in the cellular automaton procedure. The CA uses a square lattice with $N=L \times L$ cells. To overcome problems with the stoichiometry of the chemical reaction, the lattice is divided into Margolus blocks containing $2 \times 2$ cells as elementary cells. All the cells in the Margolus block are simultaneously updated following a probabilistic rule. After one sweep through the lattice, the Margolus block is shifted by one cell to the right, then down, then left, and then up to get all the different configurations. In the Mai-von Niessen model the transition probabilities of the CA depend on the initial state of the cells, on the statistical weights of the individual configurations, which are taken as classical, and on the mole fractions of the gaseous reactants, $y_{\mathrm{CO}}$ and $y_{\mathrm{O}}$, where $y_{\mathrm{O}}=1-y_{\mathrm{CO}}$. CA transition rules are shown in Fig. 1. For each initial state, the sum of all transition probabilities is normalized to 1 . In our model, $y_{\mathrm{CO}}$ is defined as the relative rate of potentially efficacious collisions of $\mathrm{CO}$ molecules on the surface; that is, $y_{\mathrm{CO}}=k_{1} /\left(k_{1}\right.$ $\left.+k_{2}\right)$, meanwhile $y_{\mathrm{O}}=k_{2} /\left(k_{1}+k_{2}\right)$. According to the ZGB model, the probability of reaction between pairs $\mathrm{CO}-\mathrm{O}$, process (3), is assumed to be unity.

\section{RESULTS}

Initially the problem was complicated, since describing the reaction requires many parameters. In this paper, we want to compare the cellular automata simulations with the results

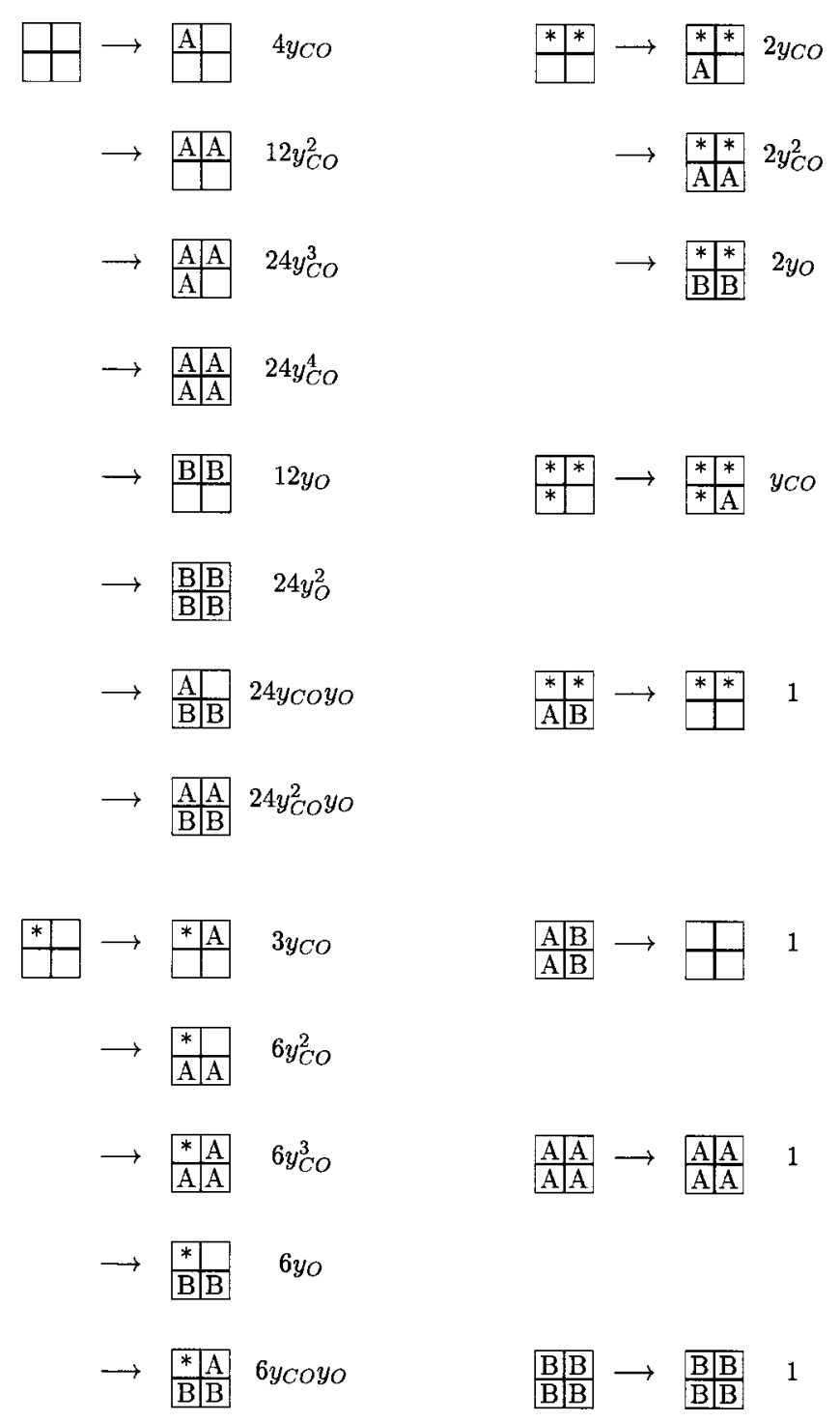

FIG. 1. Cellular automata transition rules for the $\mathrm{CO}+\mathrm{O}_{2}$ reaction. $A$ denotes a $\mathrm{CO}$ molecule, $B$ an oxygen atom, and $\star$ any occupied site. The transition probabilities of CA depend on the initial state of the cells, on the statistical weights of the individual configurations, which are taken as classical, and on the mole fractions of the gaseous reactants, $y_{\mathrm{CO}}$ and $y_{\mathrm{O}}$, where $y_{\mathrm{O}}=1-y_{\mathrm{CO}}$. In the model, $y_{\mathrm{CO}}$ is defined as $y_{\mathrm{CO}}=k_{1} /\left(k_{1}+k_{2}\right)$, where $k_{i}$ $=A_{i} \exp \left(-E_{i} / k_{B} T\right)$. For each initial state, the sum of all transition probabilities is normalized to 1 .

previously analyzed by us, starting from the kinetic equations obtained by using a mean-field approximation type.

According to experimental data, it seems that oscillations occur under the conditions where oxygen adsorption is the main mechanism. Therefore, we assume that the adsorption of $\mathrm{CO}$ and the reaction between $\mathrm{O}$ and $\mathrm{CO}$ on the surface are unactivated processes, $E_{1}=0, E_{3}=0$; the adsorption of $\mathrm{O}_{2}$ is the only activated process. We make a minor simplification considering that the reaction heat for the adsorption of $\mathrm{O}$ is twice the value of that of the $\mathrm{CO}$ and the reaction heat for $\mathrm{CO}_{2}$ is negligible, and so $h_{2}=2 h_{1}$ and $h_{3}=0$. We have fixed the values $A_{1}=0.05, A_{2}=4 \times 10^{5}, A_{3}=1, E_{1}$ $=0, E_{2} / k_{B}=6000, E_{3}=0, h_{1}=150, h_{2}=300$, and $h_{3}=0$ (in arbitrary units), which have been used in Ref. 12, where the temperature relaxation parameter $\gamma$ is the control parameter. 
The simulation was started with an empty lattice of 256 $\times 256$ sites with periodic boundary conditions. In most cases the regimes have been reached after 2000 time steps. However, for the transitions from aperiodic regime to quasiperiodic behavior and from this to poisoning of the surface by CO, 12000 and 6000 time steps were necessary, respectively. To assure the validity of our simulations we have used four different series of random numbers in each simulation, yielding essentially the same results.

For each value of $\gamma$, the simulation consisted of 2000 iterations over the entire lattice. After one sweep through the lattice, the Margolus block is shifted by one cell to the right, then down, then left, and finally up. Therefore, an iteration (time step) consists of four sweeps over the complete lattice. After each iteration, the number of adsorption processes of $\mathrm{CO}$ and $\mathrm{O}$, and the number of Langmuir-Hinshelwood events were counted and the surface temperature was modified according to Eq. (4). The data output show the temperature of the catalyst surface, $T$, the coverages fractions of $\mathrm{CO}$ and $\mathrm{O}$, $n_{\mathrm{CO}}$ and $n_{\mathrm{O}}$, and the number of pairs $\mathrm{CO}-\mathrm{O}$ which are formed per unit area, $R$, in each iteration over the entire lattice.

Oscillating regimes are obtained in the transition region where the surface changes from a complete coverage by oxygen to a complete coverage by $\mathrm{CO}$ molecules. The poisoning of the surface of the catalyst by $\mathrm{CO}$ or $\mathrm{O}$ occurs at room temperature, $T_{B}=300 \mathrm{~K}$. Depending on the temperature relaxation parameter $\gamma$, we obtain a region where the catalyst is not poisoned: $0.005 \leqslant \gamma \leqslant 0.27$. In this range there is either an aperiodic regime between the limits $0.005 \leqslant \gamma \leqslant 0.09$ or a quasiperiodic behavior for $0.10 \leqslant \gamma \leqslant 0.27$. In both cases, the surface temperature fluctuates around a value greater than ambient temperature, favoring in this way the adsorption of $\mathrm{O}_{2}$ and decreasing the adsorption of $\mathrm{CO}$. This fact shows that the $\mathrm{CO}_{2}$ production is favored. On the other hand, for $0<\gamma \leqslant 0.004$, the surface is quickly poisoned by atoms of $\mathrm{O}$ at room temperature and, therefore, the reaction between $\mathrm{CO}$ molecules and $\mathrm{O}$ atoms is not possible. The same situation happens for $\gamma \geqslant 0.28$, where the surface is covered slowly with $\mathrm{CO}$, thus producing poisoning of the catalyst by this reactant.

Our CA model is able to describe a richer variety of behaviors (quasiperiodic, aperiodic, and two poisoned states) than within the mean-field approximation. There, and for all values of $\gamma$, there was a state corresponding to complete coverage with $\mathrm{CO}$ at room temperature, which is a stable node. Besides this state, a new solution was obtained via mean-field approximation when the values of $\gamma$ varied. This new state can be either a periodic oscillatory state in the range $0.10 \leqslant \gamma \leqslant 0.15$ (the temperature fluctuates around a greater value than ambient) or a stable node for $0.03 \leqslant \gamma \leqslant 0.09$, corresponding to a reactive steady state producing $\mathrm{CO}_{2}$ at a greater temperature than room temperature. Quasiperiodic, aperiodic, and O-poisoned states were never obtained by mean-field approximation.

To study qualitatively the system behavior, we use the Poincaré's map, also known as the iterative map. If the behavior is periodic with a single frequency, the Poincaré's map will show a single point. On the other hand, if the be- havior is aperiodic, the points will appear on the map in a scattered way, not following any recognized line and filling up the iterative map. Finally, if the behavior is quasiperiodic, the points densely populate an invariant closed curve.

Figure 2 shows the time series of $T, n_{\mathrm{CO}}, n_{\mathrm{O}}$, and $R$ for two values of $\gamma$. It is also shown the corresponding iterative map for each variable. For $\gamma=0.20$, the Poincarés map shows a quasiperiodic behavior: the points are put on a same closed curve perfectly defined. However, for $\gamma=0.01$, the aperiodic behavior can be observed.

Quasiperiodic behavior of the oscillations of the catalyst temperature, coverage fractions of $\mathrm{CO}$ and $\mathrm{O}$, and $\mathrm{CO}_{2}$ production for $\gamma=0.20$ are shown in Fig. 3. Different frequencies of the quasiperiodic regime can be observed.

Between $0.10 \leqslant \gamma \leqslant 0.27$, corresponding to quasiperiodicity, the oscillation amplitude and the size of the iterative map increase as $\gamma$ increases. This is shown in Fig. 4, where it can be seen that the average production of $\mathrm{CO}_{2}$ is favored as $\gamma$ increases. At $\gamma=0.28$, the quasiperiodic regime disappears and the surface remains poisoned by $\mathrm{CO}$ at $T_{B}=300 \mathrm{~K}$.

Finally, Fig. 5 shows the four regimes which are found in the CA model. For $\gamma=0.004$, the catalyst is saturated by adsorbed oxygen after a transient time. The state obtained for $\gamma=0.07$ is an aperiodic state where the temperature of the surface fluctuates around a greater value than room temperature and a small production of $\mathrm{CO}_{2}$ is obtained due to that the surface is not saturated by any of the reactive species. For $\gamma=0.15$, the oscillatory behavior is quasiperiodic and the amplitude of the oscillations is greater than the previous state $(\gamma=0.07)$, thus increasing the averages of the densities of $\mathrm{CO}$ and $\mathrm{O}$, the yield of $\mathrm{CO}_{2}$, and the surface temperature. For $\gamma=0.28$, the surface is covered slowly with $\mathrm{CO}$, thus producing poisoning of the catalyst by this reactant. It can be also observed that the amplitude of the oscillations starts to decrease slowly until the catalyst surface is poisoned suddenly by $\mathrm{CO}$ molecules at ambient temperature.

\section{CONCLUSIONS}

In short, we have presented a CA model, extension from the Mai-von Niessen model, ${ }^{1}$ that includes the temperature as a dynamic variable with the purpose to model the oscillatory behavior of reaction of $\mathrm{CO}$. The nonlinear dependence of the rate constants on the temperature given by the Arrhenius law introduced in the CA model gives rise to quasiperiodic and aperiodic behaviors for certain values of the temperature relaxation parameter. As in others models, this parameter plays an important role in the onset of oscillatory regimes.

We think that the flexibility of the CA model presented here allows us to continue studying this surface's chemical reaction. Future works will analyze the effects of the addition of the Eley-Rideal step, the inclusion of defects on the surface, and the Langmuir-Hinshelwood as an activated process, among others topics. 

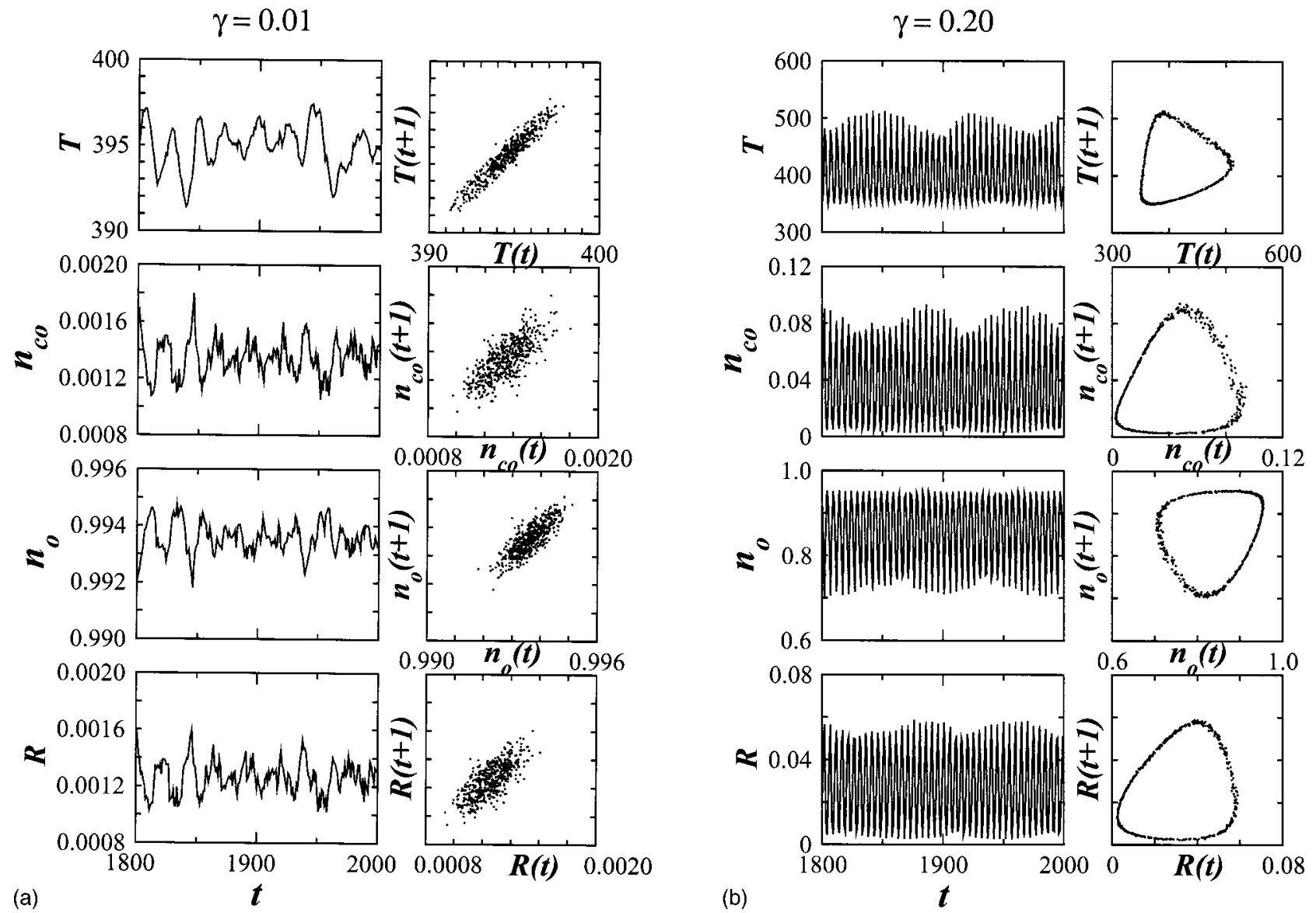

FIG. 2. Time series of the surface temperature, $T$ (in Kelvin), dimensionless coverage fractions of $\mathrm{CO}$ and $\mathrm{O}, n_{\mathrm{CO}}$ and $n_{\mathrm{O}}$, and the production of $\mathrm{CO}_{2}, R$ [defined as the number of process of type (3) which take place per unit area], for two values of the control parameter $\gamma$. The time series of the CA simulations show aperiodic and quasiperiodic regimes. (a) For $\gamma=0.01$, the regime is aperiodic as can be observed in "filled space" from the Poincaré's maps. (b) For $\gamma=0.20$, the Poincaré's maps show a quasiperiodic behavior.

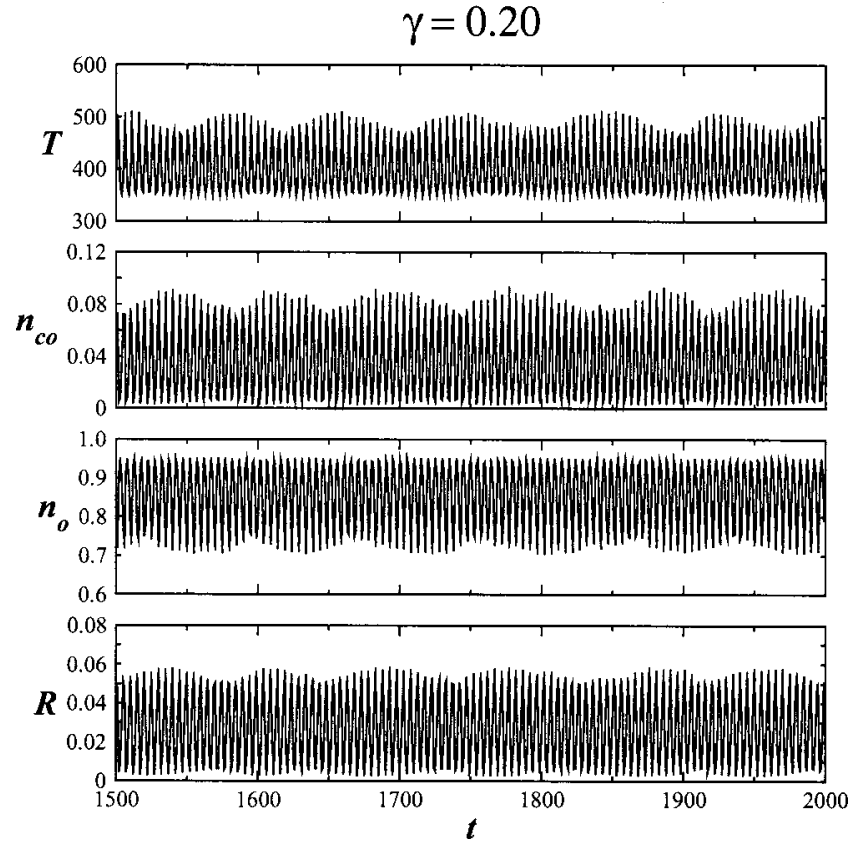

FIG. 3. Temporal behavior of the surface temperature (in kelvins), densities of $\mathrm{CO}$ and $\mathrm{O}$, and production of $\mathrm{CO}_{2}$ for $\gamma=0.20$. This state corresponds to the region where the system behavior is quasiperiodic.
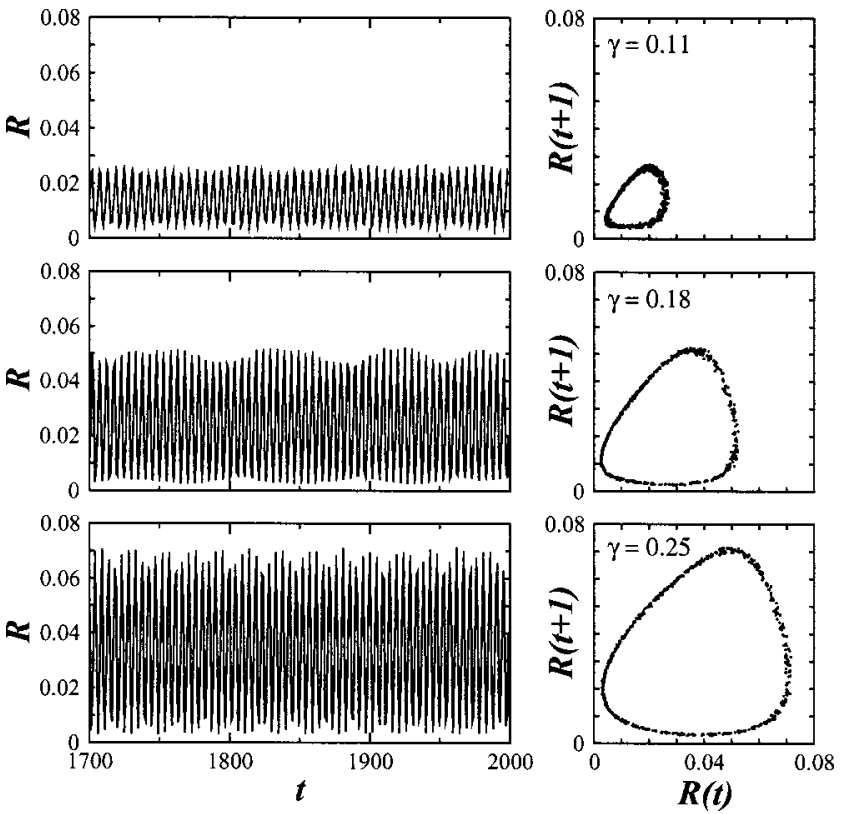

FIG. 4. Development of the oscillations of the reaction product $\mathrm{CO}_{2}$ and the associated Poincaré's maps for different values of $\gamma$. As can be seen, the oscillation amplitude and the size of the Poincaré's map increase as $\gamma$ increases, thus favoring the average yield of $\mathrm{CO}_{2}$. 

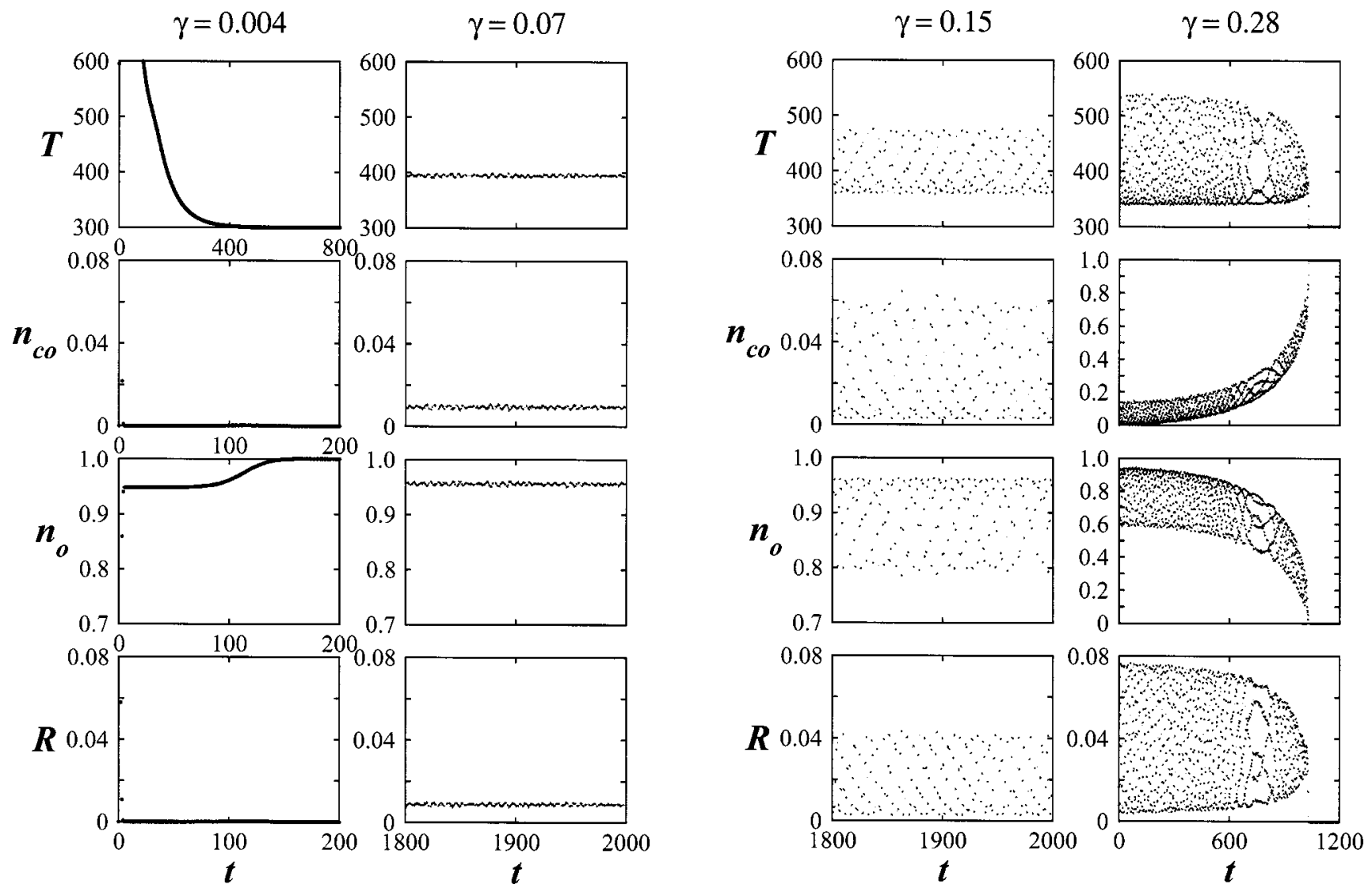

FIG. 5. Results obtained from cellular automaton simulations as a function of the control parameter $\gamma$. The graphs show the temporal behavior of the system. (a) For $\gamma=0.004$, the surface is poisoned quickly by atoms of O. For $\gamma=0.07$, there is an aperiodic state. (b) For $\gamma=0.15$, the behavior of the system is quasiperiodic and, finally, for $\gamma=0.28$, the surface is poisoned by CO. In the poisoned states, the catalyst surface remains at room temperature, $T_{B}$ $=300 \mathrm{~K}$, while in the oscillatory regimes the surface temperature fluctuates around a temperature greater than $T_{B}$.

\section{ACKNOWLEDGMENTS}

This work was partially financed by the Spanish Government (Project No. BFM2003-03986/FISI) and the European Fund for Regional Development.

${ }^{1}$ J. Mai and W. von Niessen, Phys. Rev. A 44, R6165 (1991).

${ }^{2}$ L. F. Razon and R. A. Schmitz, Catal. Rev. - Sci. Eng. 28, 89 (1986).

${ }^{3}$ S. K. Scott, Chemical Chaos (Clarendon Press, Oxford, 1991).

${ }^{4}$ M. M. Slin'ko and N. I. Jaeger, in Oscillating Heterogeneous Catalytic Systems, Studies in Surface Science and Catalysis Vol. 86, edited by B. Delmon and J. T. Yates (Elsevier, Amsterdam, 1994).
${ }^{5}$ R. E. Lagos, B. C. Sales, and H. Suhl, Surf. Sci. 82, 525 (1979).

${ }^{6}$ J. P. Dauchot and J. Van Cakenberghe, Nature (London), Phys. Sci. 246, 61 (1973); Jpn. J. Appl. Phys., Suppl. 2, 533 (1974).

${ }^{7}$ R. Dagonnier, M. Dumont, and J. Nuyts, Surf. Sci. 95, L217 (1980).

${ }^{8}$ R. E. Lagos, B. C. Sales, and H. Suhl, Surf. Sci. 95, L223 (1980).

${ }^{9}$ R. E. Lagos, T. Simoes, and A. L. Godoy, Physica A 257, 401 (1998).

${ }^{10}$ R. M. Ziff, E. Gulari, and Y. Barshad, Phys. Rev. Lett. 56, 2553 (1986).

${ }^{11}$ J. J. Luque, Phys. Rev. A 42, 3319 (1990).

${ }^{12}$ M. C. Lemos, J. J. Luque, and F. Jiménez-Morales, Phys. Rev. E 51, 5360 (1995).

${ }^{13}$ T. Toffoli and N. Margolus, Cellular Automata Machines (MIT, Moscow, 1987).

${ }^{14}$ S. S. Tambe, V. K. Jayaraman, and B. D. Kulkarni, Chem. Phys. Lett. 225, 303 (1994). 